\title{
Clinicopathological and prognostic significance of sialyl Lewis $X$ overexpression in patients with cancer: a meta-analysis
}

This article was published in the following Dove Press journal:

OncoTargets and Therapy

24 May 2016

Number of times this article has been viewed

\author{
Jin-xiao Liang' \\ Yong Liang ${ }^{2}$ \\ Wei $\mathrm{Gao}^{3}$
}

'Department of Thoracic Surgery, Zhejiang Cancer Hospital, Hangzhou, People's Republic of China; ${ }^{2}$ Department of Clinical Medicine, Taizhou University Medical School, Taizhou, People's Republic of China; ${ }^{3}$ School of Medicine, Zhejiang University City College, Hangzhou, Zhejiang, People's Republic of China

Correspondence: Wei Gao School of Medicine, Zhejiang University City College, 48 Huzhou Street, Hangzhou, Zhejiang, People's Republic of China

Tel +86 I58 69027992

Fax +86 57। 88I22508

Email gaoweizucc_1986@163.com

\begin{abstract}
Many studies have shown that sialyl Lewis $\mathrm{X}\left(\mathrm{sLe}^{\mathrm{X}}\right)$ is related to cancer prognosis and clinicopathology, but failed to provide conclusive results. We conducted the present meta-analysis to identify the association between $\mathrm{sLe}^{\mathrm{X}}$ overexpression and cancer prognosis. We searched studies in PubMed and Embase databases. Relative risk or hazard ratio with 95\% confidence intervals were estimated with the Mantel-Haenszel random-effect method and 29 studies were included. Our meta-analysis showed that $\mathrm{sLe}^{\mathrm{x}}$ overexpression is significantly related to lymphatic invasion, venous invasion, $\mathrm{T}$ stage, $\mathrm{N}$ stage, $\mathrm{M}$ stage, tumor stage, recurrence, and overall survival. In subgroup analysis, we found that cancer type and ethnicity might be two major contributing factors to the possible presence of heterogeneity among the studies. In conclusion, $\mathrm{sLe}^{\mathrm{X}}$ overexpression is associated with tumor metastasis, recurrence, and overall survival in cancer patients, it plays an important role in cancer prognosis.
\end{abstract}

Keywords: sialyl Lewis X, cancer, prognosis, meta-analysis

\section{Introduction}

As is known to all, cancer is a common life-threatening disease. According to recent studies, the incidence of cancer increases 1\% per year in Europe. ${ }^{1}$ Among the adult population, a rising trend is reported for soft tissue sarcoma. ${ }^{2}$ Breast, colorectal, prostate, and lung cancers are the most common oncological cause for death among the European population. ${ }^{3}$ Cancer cannot be cured, as expected, due to the limited knowledge of iatrotechnique. So, exploration of more precise bio-indicators is valuable for early diagnosis of cancer and improving prognosis of patients.

Cell surface carbohydrates are involved in various biological processes such as cellular differentiation, maturation, proliferation, and malignant transformation. ${ }^{4}$ Dramatic changes of cell surface carbohydrates are associated with cancer occurrence, tumor invasiveness, and metastatic behavior. ${ }^{5}$ Sialyl Lewis X $\left(\mathrm{sLe}^{\mathrm{X}}\right)(\mathrm{NeuNAc} \alpha 2,3 \mathrm{Gal} \beta 1,4[\mathrm{Fuc} \alpha 1,3]$ GlcNAc), a carbohydrate antigen, is related to cell adhesion and our previous study showed that inhibition of $\mathrm{sLe}^{\mathrm{x}}$ synthesis leads to decreased adhesion of trophoblast cells to endometrial epithelial cells. ${ }^{6}$ Also, $\mathrm{sLe}^{\mathrm{X}}$ is frequently expressed in human cancer cells and primary tumors. ${ }^{7,8}$ As a ligand for E-selectin and L-selectin, $\mathrm{sLe}^{\mathrm{X}}$ is related to cell adhesion. ${ }^{9}$ It has been demonstrated that $\mathrm{sLe}^{\mathrm{X}}$ was involved in the adhesion of tumor cells to vascular endothelium. ${ }^{10}$ The potential role of $\mathrm{sLe}^{\mathrm{x}}$ in the tumor metastatic process has been supported by several clinical studies. ${ }^{11-14}$

Many studies have identified the relationship between $\mathrm{sLe}^{\mathrm{x}}$ and cancer prognosis, but individual studies of the influence of $\mathrm{sLe}^{\mathrm{X}}$ expression in cancer have failed to 
provide conclusive results. The present meta-analysis was conducted to further explore the relationship between $\mathrm{sLe}^{\mathrm{X}}$ expression and cancer prognosis and clinicopathology.

\section{Materials and methods}

\section{Publication search}

We searched published studies in the PubMed and Embase databases up to May 2014 with the following search terms: (slex OR sialyl lewis x) AND (cancer OR neoplasms OR carcinoma OR tumor) AND prognosis. Furthermore, reference lists of main reports and review articles were also reviewed to identify additional relevant publications. The study was conducted and reported following the PRISMA (Preferred Reporting Items for Systematic Reviews and Meta-Analyses) guidelines.

\section{Selection criteria}

Two authors (YL and JXL) reviewed the retrieved titles and abstracts to discriminate the eligible studies for inclusion in our meta-analysis independently. Published studies were included based on the following criteria: 1) written and published in English; 2) patients with cancer diagnosis by pathology; 3) studies about $\mathrm{SLe}^{\mathrm{x}}$ expression in cancer tissues; 4) $\mathrm{SLe}^{\mathrm{X}}$ expression was measured by immunohistochemistry (IHC) method; 5) full length paper with sufficient data on $\mathrm{sLe}^{\mathrm{x}}$ expression and prognosis and prognosis-related factors; 6 ) we could find the full text. We excluded studies with the following criteria: 1) written and published in a language other than English; 2) studies about cell lines and animals; 3) studies about $\mathrm{sLe}^{\mathrm{X}}$ expression in serum; 4) review articles without original data; 5) a commentary, letter to the editor, or monograph.

\section{Data extraction}

Two authors (YL and WG) performed the data evaluation independently. The following data were extracted from each study: the first author's last name; publication year; country; cancer source; number of patients; number of $\mathrm{sLe}^{\mathrm{X}}$ expressions (positive/negative); clinicopathological factors (age, sex, tumor size, histological differentiation, lymphatic invasion, venous invasion, T/N/M stage, tumor stage, and recurrence); survival analysis.

\section{Data synthesis and statistical analysis}

Expression of $\mathrm{sLe}^{\mathrm{x}}$ was analyzed as dichotomous variables, as positive expression versus negative expression. The clinicopathological factors were also conducted as dichotomous variables, as older age versus younger age for age; male versus female for sex; large versus small for tumor size; high versus low for histological differentiation; I and II versus III and IV for tumor stage; pT2 versus more than pT3 for depth of invasion (T stage); with versus without for lymphatic invasion, venous invasion, lymph node metastasis (N stage), distant metastasis (M stage), recurrence. Survival of $\mathrm{SLe}^{\mathrm{X}}$ expression was analyzed by Cox's regression analysis conducted as hazard ratio (HR) and 95\% confidence interval (95\% CI). The data of expression of $\mathrm{sLe}^{\mathrm{x}}$ and clinicopathological factors or survival rate were extracted and calculated by initial data of studies. These data were analyzed with random-effect method, and were measured in relative risk (RR) with $95 \%$ CI. Statistical heterogeneity was estimated by means of Cochran's Q test and $I^{2}$ test. The $I^{2}$ test represents the percentage of variation to heterogeneity, which is categorized as low $(0 \%-40 \%)$, moderate $(40 \%-60 \%)$, high $(60 \%-90 \%)$, very high $(>90 \%)$. Subgroup analyses were carried out based on cancer or country of the included studies if a significant heterogeneity was found in overall meta-analysis. To identify any potential publication bias, we used Begg's test. All statistical analyses were performed with Review Manager 5.2 and STATA 12.0.

\section{Results}

\section{Systematic review}

We identified 178 studies that fit our search strategy, 41 studies were identified in our primary search (Figure 1). Finally, 29 studies published between 1993 and 2013 were included in our meta-analysis..$^{11,12,14} 40$ Detailed characteristics of these studies are provided in Table 1.

\section{Association of $s L e^{X}$ expression with cancer prognosis and clinicopathology}

$\mathrm{sLe}^{\mathrm{x}}$ expression correlated with prognostic factors, including lymphatic invasion (lymphatic invasion versus nonlymphatic invasion) (pooled RR $=1.36,95 \%$ CI: $1.15-1.61$, $I^{2}=62.3 \%$ ), venous invasion (venous invasion versus nonvenous invasion) (pooled $\mathrm{RR}=1.41,95 \% \mathrm{CI}: 1.18-1.67$, $\left.I^{2}=52.9 \%\right)$, T stage (pT3-4 stage versus pT2 stage) (pooled $\mathrm{RR}=1.14,95 \%$ CI: $1.04-1.27, I^{2}=59.6 \%$ ), N stage (lymph node metastasis versus non-lymph node metastasis) (pooled RR $=1.46,95 \%$ CI: $1.29-1.66, I^{2}=55.1 \%$ ), M stage (distant metastasis versus non-distant metastasis) (pooled $\left.\mathrm{RR}=1.76,95 \% \mathrm{CI}: 1.34-2.31, I^{2}=42.1 \%\right)$, tumor stage (stage III/IV versus stage I/II) (pooled RR $=1.42,95 \% \mathrm{CI}$ : $1.19-1.68, I^{2}=69.9 \%$ ), tumor recurrence (recurrence versus non-recurrence) (pooled $\mathrm{RR}=2.92,95 \% \mathrm{CI}: 2.02-4.23$, $\left.I^{2}=0.0 \%\right)($ Figure $2 \mathrm{~A})$. 


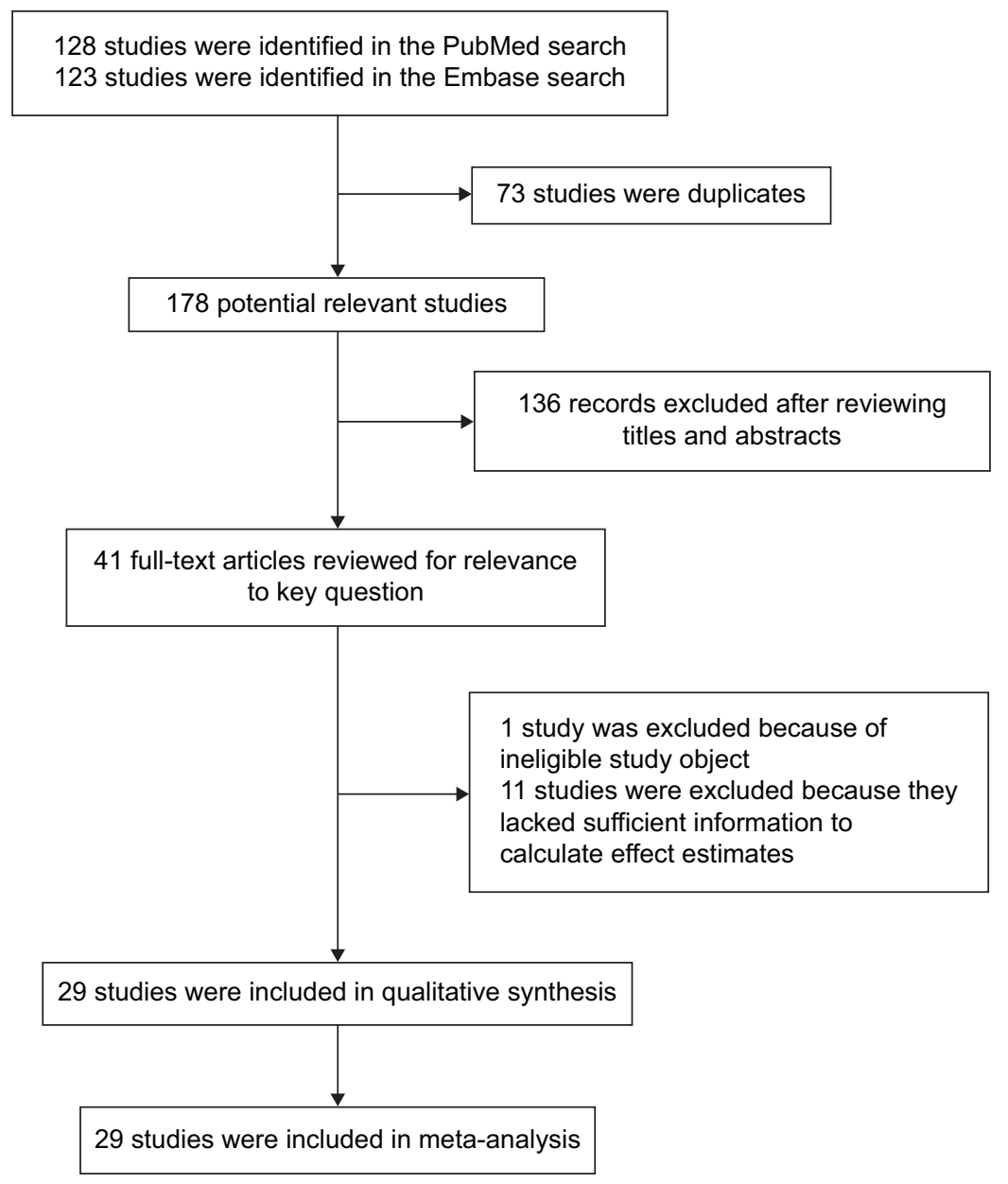

Figure I The flow diagram of included/excluded studies.

Meantime, we found that $\mathrm{sLe}^{\mathrm{x}}$ overexpression was not significantly related to cancer prognosis and clinicopathology factors, including age (older versus younger) (pooled $\mathrm{RR}=1.08,95 \%$ CI: $0.97-1.21, I^{2}=0.0 \%$ ), sex (male versus female) (pooled RR $=0.97,95 \%$ CI: $0.88-1.07, I^{2}=47.0 \%$ ), tumor size (larger versus smaller) (pooled $\mathrm{RR}=1.23,95 \%$ CI: $0.94-1.62, I^{2}=51.1 \%$ ), tumor differentiation (lower differentiation versus higher differentiation) (pooled $R R=0.94$, 95\% CI: 0.72-1.21, $P^{2}=75.1 \%$ ) (Figure 2B).

\section{sLe ${ }^{X}$ overexpression on cancer survival}

Eight studies analyzed the overall survival (OS) of human cancer with positive/negative $\mathrm{sLe}^{\mathrm{X}}$ overexpression, the HRs ranged from 2.42 to $9.10 .{ }^{18,30,32,34-36,38,39}$ The summarized HR of negative versus positive was 3.11 (95\% CI: 2.25-4.32) with low heterogeneity $\left(I^{2}=0.0 \%\right)$ (Figure 3 ).

\section{Subgroup analyses}

We chose subgroup analyses in meta-analysis with relative high heterogeneity $\left(I^{2}>40 \%\right)$. In subgroup analyses, studies were stratified by cancer category (colorectal cancer, gastric cancer, lung cancer, breast cancer, head and neck squamous cell carcinoma, esophageal squamous cell carcinoma, oral squamous cell carcinoma, gallbladder cancer, pancreatic ductal adenocarcinoma, prostate cancer, and extrahepatic bile duct carcinoma) or ethnicity (Asia, America, and Europe). In addition, most of these analyses showed low heterogeneity after stratification (Tables 2 and 3).

\section{Publication bias}

Begg's test was created for assessment of possible publication bias. It suggested that publication bias had little influence on these meta-analysis results $(P>0.05)$ (Figure 4).

\section{Discussion}

The cancer statistics of the USA, in $2013,{ }^{41}$ clearly indicated that the methods of treatment for cancer need to be improved. Exploring new molecular biological prognostic and predictive markers is a hot topic in modern medicine. Nakagoe et al first reported that $\mathrm{sLe}^{\mathrm{X}}$ was expressed in serum 
Table I Characteristics of the included studies

\begin{tabular}{|c|c|c|c|c|c|c|}
\hline Study ID & Country & $\begin{array}{l}\text { Cancer } \\
\text { source }\end{array}$ & $\begin{array}{l}\text { Number } \\
\text { of patients }\end{array}$ & $\begin{array}{l}\text { sLe }{ }^{\mathrm{x}} \text { expression } \\
\text { (positive/negative) }\end{array}$ & Clinicopathological factors & $\begin{array}{l}\text { Survival } \\
\text { analysis }\end{array}$ \\
\hline $\begin{array}{l}\text { Nakamori et al }{ }^{18} \\
(1993)\end{array}$ & Japan & $\begin{array}{l}\text { Colorectal } \\
\text { cancer }\end{array}$ & 132 & $50 / 82$ & $\begin{array}{l}\text { Sex, differentiation, } \mathrm{T} \text { stage, } \mathrm{N} \text { stage, } \\
\text { lymphatic invasion, venous invasion, } \\
\text { tumor stage, recurrence }\end{array}$ & NA \\
\hline $\begin{array}{l}\text { Yamaguchi et al } \\
\text { (1994) }\end{array}$ & Japan & $\begin{array}{l}\text { Colorectal } \\
\text { cancer }\end{array}$ & 170 & $56 / 114$ & $\begin{array}{l}\text { Differentiation, } \mathrm{T} \text { stage, } \mathrm{N} \text { stage, } \\
\text { lymphatic invasion, venous invasion, } \\
\text { tumor stage, recurrence }\end{array}$ & NA \\
\hline Idikio $^{20}(1997)$ & Canada & Prostate cancer & 38 & $30 / 8$ & Differentiation & NA \\
\hline Nakamori et $\mathrm{al}^{21}$ (1997) & Japan & $\begin{array}{l}\text { Colorectal } \\
\text { cancer }\end{array}$ & 159 & $58 / 101$ & $\begin{array}{l}\text { Age, sex, differentiation, } \mathrm{T} \text { stage, } \\
\mathrm{N} \text { stage, lymphatic invasion, venous } \\
\text { invasion, tumor stage }\end{array}$ & NA \\
\hline $\begin{array}{l}\text { Shimodaira et } \mathrm{al}^{22} \\
\text { (1997) }\end{array}$ & Japan & $\begin{array}{l}\text { Colorectal } \\
\text { cancer }\end{array}$ & 43 & $28 / 15$ & $\begin{array}{l}\text { Tumor size, differentiation, } \mathrm{T} \text { stage, } \\
\mathrm{N} \text { stage, lymphatic invasion, venous } \\
\text { invasion, tumor stage }\end{array}$ & NA \\
\hline Ura et al ${ }^{12}(1997)$ & Japan & Gastric cancer & 110 & $91 / 19$ & T stage, $\mathrm{N}$ stage & NA \\
\hline Baldus et al ${ }^{17}(1998)$ & Germany & Gastric cancer & 127 & $85 / 42$ & Sex, tumor stage & NA \\
\hline Farmer et $\mathrm{a}^{23}(1998)$ & United States & HNSCC & 82 & $5|/ 3|$ & Age, sex, $M$ stage, tumor stage & NA \\
\hline Fukuoka et al" (1998) & Japan & Lung cancer & 52 & $34 / 18$ & $\mathrm{~N}$ stage, $\mathrm{M}$ stage & NA \\
\hline Tatsumi et al ${ }^{24}$ (1998) & Japan & Gastric cancer & 87 & $41 / 46$ & $\begin{array}{l}\text { Differentiation, } T \text { stage, } \mathrm{N} \text { stage, } \\
M \text { stage, lymphatic invasion, venous } \\
\text { invasion }\end{array}$ & NA \\
\hline Yamaguchi et $\mathrm{al}^{25}$ (1998) & Japan & Breast cancer & 102 & $61 / 41$ & Age, tumor size, $\mathrm{N}$ stage & NA \\
\hline Kurahara et al ${ }^{14}$ (1999) & Japan & OSCC & 70 & $24 / 46$ & M stage & NA \\
\hline Takao et a ${ }^{26}(1999)$ & Japan & EBDC & 73 & $45 / 28$ & $\begin{array}{l}\text { Age, sex, differentiation, } \mathrm{T} \text { stage, } \\
\mathrm{N} \text { stage, } \mathrm{M} \text { stage, lymphatic invasion, } \\
\text { venous invasion, tumor stage }\end{array}$ & NA \\
\hline Futamura et al ${ }^{27}(2000)$ & Japan & Gastric cancer & 245 & $135 / 110$ & $\begin{array}{l}\text { Age, sex, differentiation, } \mathrm{T} \text { stage, } \\
\mathrm{N} \text { stage, } \mathrm{M} \text { stage, venous invasion, } \\
\text { tumor stage }\end{array}$ & NA \\
\hline Grabowski et $\mathrm{al}^{28}$ (2000) & Germany & $\begin{array}{l}\text { Colorectal } \\
\text { cancer }\end{array}$ & 182 & $103 / 79$ & $\begin{array}{l}\text { Sex, differentiation, } T \text { stage, } N \text { stage, } \\
M \text { stage, tumor stage }\end{array}$ & Multi \\
\hline Nakagoe et al ${ }^{16}(2000)$ & Japan & $\begin{array}{l}\text { Colorectal } \\
\text { cancer }\end{array}$ & 101 & $76 / 25$ & Tumor stage & Uni \\
\hline Machida et $\mathrm{al}^{29}(200 \mathrm{I})$ & Japan & Lung cancer & 25 & $19 / 6$ & $\begin{array}{l}\text { Tumor size, } N \text { stage, } M \text { stage, } \\
\text { lymphatic invasion, venous invasion }\end{array}$ & NA \\
\hline Takahashi et al ${ }^{30}(200 \mathrm{I})$ & Japan & PDAC & 23 & $15 / 8$ & NA & Multi \\
\hline Baldus et a ${ }^{31}(2002)$ & Germany & $\begin{array}{l}\text { Colorectal } \\
\text { cancer }\end{array}$ & 243 & $165 / 78$ & $\begin{array}{l}\text { Differentiation, } N \text { stage, } M \text { stage, } \\
\text { tumor stage }\end{array}$ & NA \\
\hline Konno et al ${ }^{32}$ (2002) & Japan & $\begin{array}{l}\text { Colorectal } \\
\text { cancer }\end{array}$ & 134 & $47 / 87$ & $\mathrm{~N}$ stage, $\mathrm{M}$ stage, venous invasion & Multi \\
\hline Nakagoe et al $\left.\right|^{34}$ (2002) & Japan & Breast cancer & 87 & $37 / 50$ & $\begin{array}{l}\text { Age, differentiation, } T \text { stage, } N \text { stage, } \\
M \text { stage, tumor stage }\end{array}$ & Multi \\
\hline Nakagoe et al $\mathrm{l}^{33,34}$ (2002) & Japan & Gastric cancer & 101 & $31 / 70$ & $\begin{array}{l}\text { Age, sex, tumor size, differentiation, } \\
\mathrm{T} \text { stage, } \mathrm{N} \text { stage, lymphatic invasion, } \\
\text { venous invasion }\end{array}$ & Multi \\
\hline Kashiwagi et al ${ }^{35}$ (2004) & Japan & $\begin{array}{l}\text { Gallbladder } \\
\text { cancer }\end{array}$ & 54 & $28 / 26$ & $\begin{array}{l}\text { T stage, } \mathrm{N} \text { stage, lymphatic invasion, } \\
\text { venous invasion }\end{array}$ & NA \\
\hline Yu et al ${ }^{36}(2005)$ & $\begin{array}{l}\text { People's Republic } \\
\text { of China }\end{array}$ & Lung cancer & 61 & $40 / 21$ & Age, sex, T stage, $N$ stage, recurrence & Uni \\
\hline Faried et $\mathrm{al}^{37}$ (2007) & Japan & ESCC & 130 & $40 / 90$ & $\begin{array}{l}\text { Sex, differentiation, } T \text { stage, } N \text { stage, } \\
M \text { stage, lymphatic invasion, venous } \\
\text { invasion, tumor stage }\end{array}$ & Multi \\
\hline Croce et $\mathrm{al}^{38}(2008)$ & Argentina & HNSCC & 125 & $29 / 96$ & $\begin{array}{l}\text { Age, sex, differentiation, } T \text { stage, } \\
N \text { stage, tumor stage }\end{array}$ & NA \\
\hline Sozzani et al ${ }^{39}(2008)$ & Italy & Breast cancer & 127 & $37 / 90$ & $\begin{array}{l}\text { Differentiation, } \mathrm{T} \text { stage, } \mathrm{N} \text { stage, } \\
\text { venous invasion }\end{array}$ & NA \\
\hline Portela et al ${ }^{40}(20 \mathrm{I} I)$ & Spain & $\begin{array}{l}\text { Colorectal } \\
\text { cancer }\end{array}$ & 155 & $67 / 88$ & $\begin{array}{l}\text { Age, sex, tumor size, differentiation, } \\
\text { T stage, } N \text { stage, } M \text { stage, tumor stage }\end{array}$ & NA \\
\hline Schiffmann et al ${ }^{15}(20 \mid 2)$ & Germany & $\begin{array}{l}\text { Colorectal } \\
\text { cancer }\end{array}$ & 215 & $102 / 113$ & $\begin{array}{l}\text { Sex, differentiation, T stage, } \mathrm{N} \text { stage, } \\
M \text { stage }\end{array}$ & NA \\
\hline
\end{tabular}

Abbreviations: NA, not available; OSCC, oral squamous cell carcinoma; EBDC, extrahepatic bile duct carcinoma; PDAC, pancreatic ductal adenocarcinoma; Multi, Multivariate; Uni, Univariate; sLe ${ }^{\mathrm{X}}$, sialyl Lewis X; ESCC, esophageal squamous cell carcinoma; HNSCC, head and neck squamous cell carcinoma. 

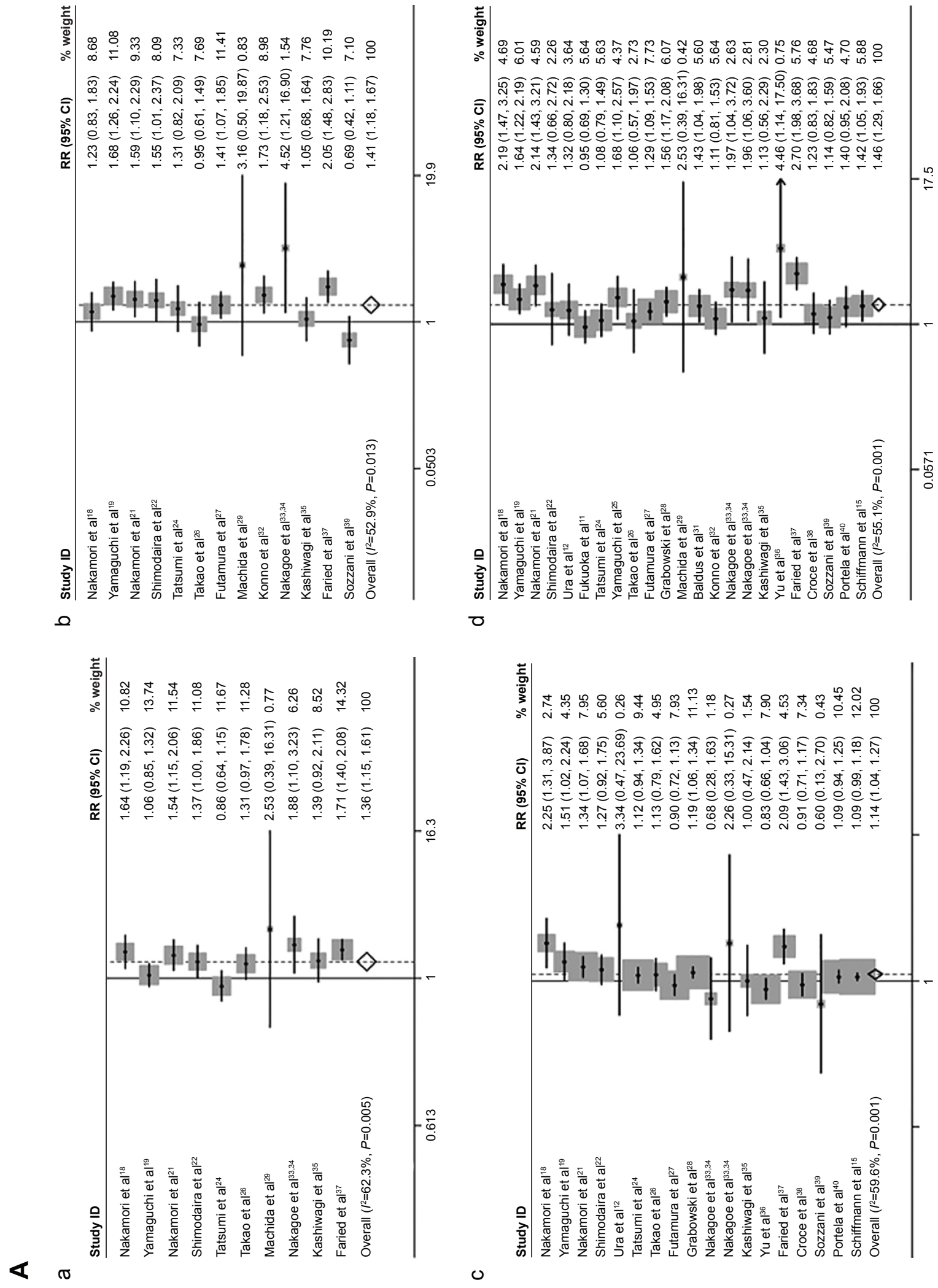

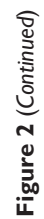




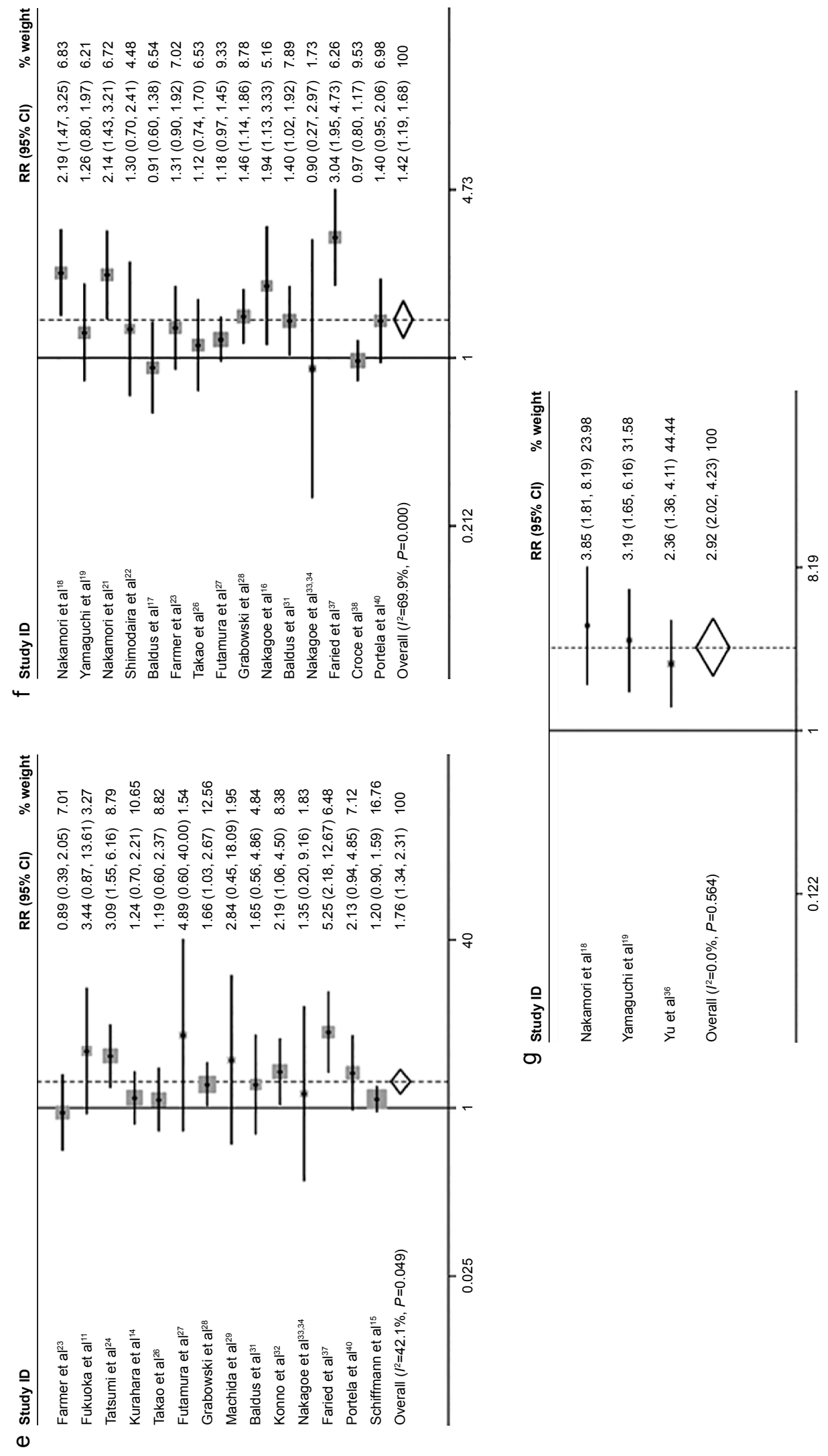



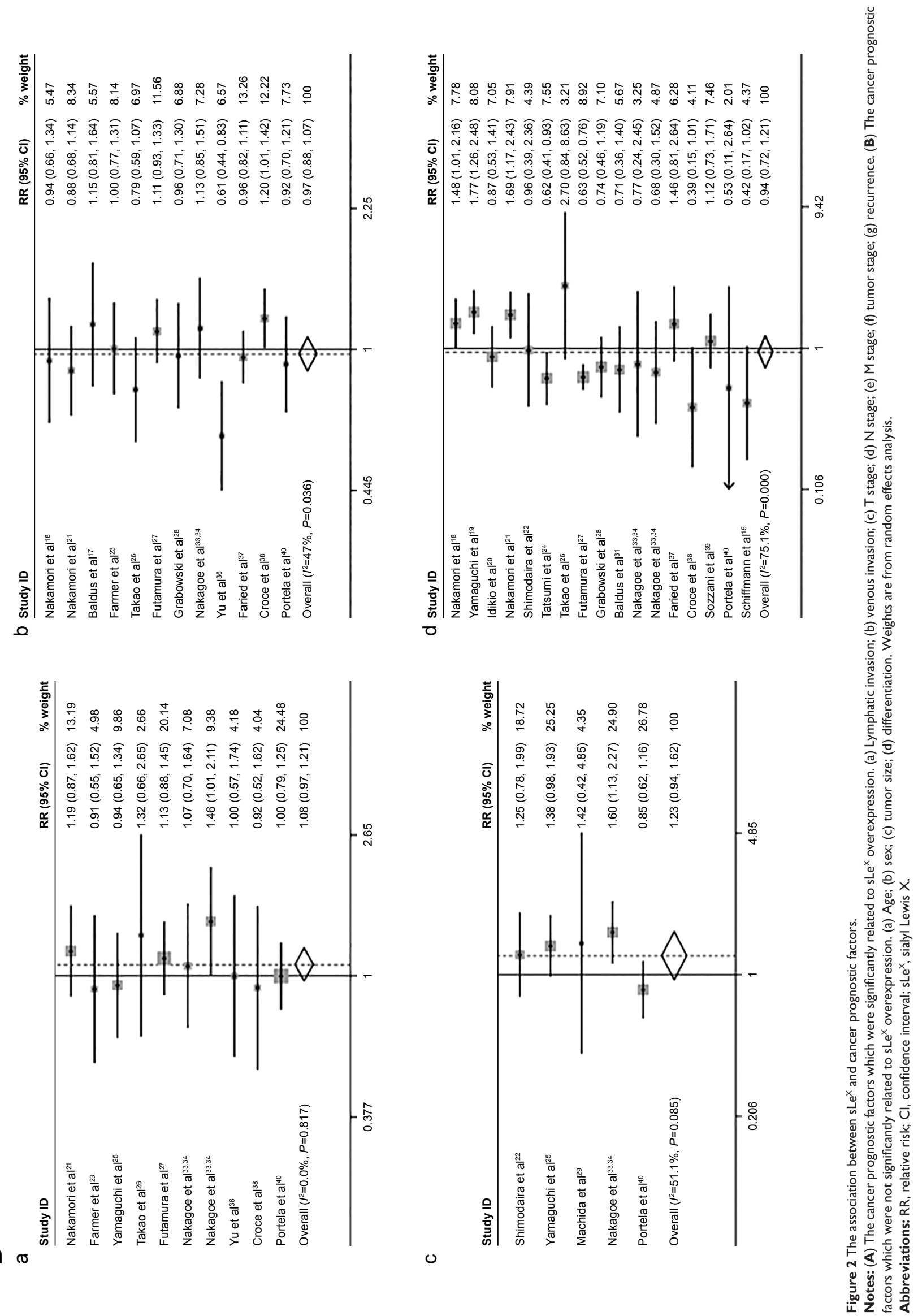


\begin{tabular}{|c|c|c|}
\hline Study ID & HR $(95 \% \mathrm{Cl})$ & $\%$ weight \\
\hline Grabowski et al ${ }^{28}$ & $3.81(1.80,7.90)$ & 19.46 \\
\hline Nakagoe et $a^{16}$ & $2.90(1.01,8.37)$ & 9.52 \\
\hline Takahashi et al ${ }^{30}$ & $5.27(1.09,25.60)$ & 4.26 \\
\hline Konno et $\mathrm{al}^{32}$ & $2.87(1.21,6.81)$ & 14.28 \\
\hline Nakagoe et al ${ }^{33,34}$ & $3.80(1.21,1.98)$ & 8.10 \\
\hline Nakagoe et al ${ }^{33,34}$ & $9.10(1.28,64.90)$ & 2.76 \\
\hline Yu et $a^{36}$ & $2.86(1.03,8.45)$ & 9.63 \\
\hline Faried et $\mathrm{al}^{37}$ & $2.42(1.36,4.31)$ & 32.00 \\
\hline Overall $\left(I^{2}=0.0 \%, P=0.904\right)$ & $3.11(2.25,4.32)$ & 100 \\
\hline 0.0154 & & \\
\hline
\end{tabular}

Figure 3 Meta-analysis with a random-effect model for the association of sLe ${ }^{x}$ overexpression with overall survival.

Note: Weights are from random effects analysis.

Abbreviations: $\mathrm{HR}$, hazard ratio; $\mathrm{Cl}$, confidence interval; sLe ${ }^{\mathrm{X}}$, sialyl Lewis $\mathrm{X}$.

Table 2 Subgroup analyses of country

\begin{tabular}{|c|c|c|c|c|}
\hline & $\begin{array}{l}\text { Number } \\
\text { of studies }\end{array}$ & $\begin{array}{l}\text { Summary RR } \\
\text { (95\% Cls) }\end{array}$ & $I^{2}$ value & $\mathbf{P}_{\mathrm{h}}$ \\
\hline \multicolumn{5}{|l|}{ Sex } \\
\hline Overall & 12 & $0.97(0.88,1.07)$ & $47.0 \%$ & 0.036 \\
\hline Asia & 7 & $0.92(0.80,1.06)$ & $56.5 \%$ & 0.032 \\
\hline Europe & 3 & $0.99(0.83,1.18)$ & $0.0 \%$ & 0.593 \\
\hline Americas & 2 & $1.13(0.95,1.34)$ & $24.2 \%$ & 0.251 \\
\hline \multicolumn{5}{|c|}{ Tumor size } \\
\hline Overall & 5 & $1.23(0.94,1.62)$ & $51.1 \%$ & 0.085 \\
\hline Asia & 4 & $1.43(1.16,1.77)$ & $0.0 \%$ & 0.853 \\
\hline Europe & I & $0.85(0.62,1.16)$ & NA & NA \\
\hline \multicolumn{5}{|c|}{ Differentiation } \\
\hline Overall & 17 & $0.94(0.72,1.21)$ & $75.1 \%$ & 0.000 \\
\hline Asia & 11 & I.II $(0.80,1.55)$ & $82.3 \%$ & 0.000 \\
\hline Europe & 4 & $0.66(0.46,0.93)$ & $0.0 \%$ & 0.715 \\
\hline Americas & 2 & $0.63(0.25,1.57)$ & $67.8 \%$ & 0.078 \\
\hline \multicolumn{5}{|c|}{ Venous invasion } \\
\hline Overall & 13 & I.4I (I.18, I.67) & $52.9 \%$ & 0.013 \\
\hline Asia & 12 & $1.49(1.29,1.72)$ & $31.0 \%$ & 0.143 \\
\hline Europe & 1 & $0.69(0.42, I . I I)$ & NA & NA \\
\hline \multicolumn{5}{|l|}{ T stage } \\
\hline Overall & 18 & $1.14(1.04,1.27)$ & $59.6 \%$ & 0.001 \\
\hline Asia & 13 & $1.23(1.03,1.47)$ & $67.5 \%$ & 0.000 \\
\hline Europe & 4 & I.II (I.05, I.19) & $0.0 \%$ & 0.497 \\
\hline Americas & I & $0.91(0.71,1.17)$ & NA & NA \\
\hline \multicolumn{5}{|l|}{ N stage } \\
\hline Overall & 23 & $1.46(1.29,1.66)$ & $55.1 \%$ & 0.001 \\
\hline Asia & 17 & $1.53(1.28,1.82)$ & $65.7 \%$ & 0.000 \\
\hline Europe & 5 & $1.40(1.21,1.61)$ & $0.0 \%$ & 0.724 \\
\hline Americas & I & $1.23(0.83,1.83)$ & NA & NA \\
\hline \multicolumn{5}{|l|}{ M stage } \\
\hline Overall & 14 & $1.76(1.34,2.31)$ & $42.1 \%$ & 0.049 \\
\hline Asia & 9 & $2.20(1.47,3.30)$ & $38.3 \%$ & 0.113 \\
\hline Europe & 4 & $1.37(1.09,1.72)$ & $0.0 \%$ & 0.410 \\
\hline Americas & I & $0.89(0.39,2.05)$ & NA & NA \\
\hline \multicolumn{5}{|c|}{ Tumor stage } \\
\hline Overall & 15 & $1.42(1.19,1.68)$ & $69.9 \%$ & 0.000 \\
\hline Asia & 9 & $1.62(1.24,2.10)$ & $69.4 \%$ & 0.001 \\
\hline Europe & 4 & $1.32(1.10,1.59)$ & $22.3 \%$ & 0.277 \\
\hline Americas & 2 & $1.08(0.79,1.49)$ & $58.7 \%$ & 0.120 \\
\hline
\end{tabular}

Note: $\mathrm{P}_{\mathrm{h}}: P$-value for heterogeneity within each subgroup.

Abbreviations: $\mathrm{RR}$, relative risk; $\mathrm{Cl}$, confidence interval; $\mathrm{NA}$, not available. 
Table 3 Subgroup analyses of cancer types

\begin{tabular}{|c|c|c|c|c|}
\hline Subgroup & $\begin{array}{l}\text { Number } \\
\text { of studies }\end{array}$ & $\begin{array}{l}\text { Summary RR } \\
\text { (95\% Cls) }\end{array}$ & $I^{2}$ value & $\mathbf{P}_{\mathrm{h}}$ \\
\hline \multicolumn{5}{|l|}{ Sex } \\
\hline Overall & 12 & $0.97(0.88,1.07)$ & $47.0 \%$ & 0.036 \\
\hline Colorectal cancer & 4 & $0.92(0.80,1.06)$ & $0.0 \%$ & 0.978 \\
\hline Gastric cancer & 3 & I.I2 (0.97, I.29) & $0.0 \%$ & 0.981 \\
\hline HNSCC & 2 & $1.13(0.95,1.34)$ & $24.2 \%$ & 0.251 \\
\hline EBDC & 1 & $0.79(0.59,1.07)$ & NA & NA \\
\hline Lung cancer & 1 & $0.6 \mathrm{I}(0.44,0.83)$ & NA & NA \\
\hline ESCC & 1 & $0.96(0.82,1.11)$ & NA & NA \\
\hline \multicolumn{5}{|l|}{ Tumor size } \\
\hline Overall & 5 & $1.23(0.94,1.62)$ & $51.1 \%$ & 0.085 \\
\hline Colorectal cancer & 2 & $0.99(0.68,1.44)$ & $46.7 \%$ & 0.171 \\
\hline Breast cancer & 1 & $1.38(0.98,1.93)$ & NA & NA \\
\hline Lung cancer & 1 & I.42 $(0.42,4.85)$ & NA & NA \\
\hline Gastric cancer & 1 & $1.60(1.13,2.27)$ & NA & NA \\
\hline \multicolumn{5}{|l|}{ Differentiation } \\
\hline Overall & 17 & $0.94(0.72,1.2 \mathrm{I})$ & $75.1 \%$ & 0.000 \\
\hline Colorectal cancer & 8 & $1.06(0.74,1.52)$ & $69.6 \%$ & 0.002 \\
\hline Gastric cancer & 3 & $0.63(0.53,0.75)$ & $0.0 \%$ & 0.978 \\
\hline Breast cancer & 2 & $1.07(0.72,1.60)$ & $0.0 \%$ & 0.548 \\
\hline Prostate cancer & 1 & $0.87(0.53, I .4 I)$ & NA & NA \\
\hline EBDC & 1 & $2.70(0.84,8.63)$ & NA & NA \\
\hline ESCC & 1 & $\mathrm{I} .46(0.8 \mathrm{I}, 2.64)$ & NA & NA \\
\hline HNSCC & I & $0.39(0.15,1.01)$ & NA & NA \\
\hline \multicolumn{5}{|c|}{ Lymphatic invasion } \\
\hline Overall & 10 & $1.36(1.15,1.61)$ & $62.3 \%$ & 0.005 \\
\hline Colorectal cancer & 4 & $1.36(1.09,1.68)$ & $56.7 \%$ & 0.074 \\
\hline Gastric cancer & 2 & I.23 $(0.55,2.73)$ & $85.4 \%$ & 0.009 \\
\hline EBDC & 1 & I.3I (0.97, I.78) & NA & NA \\
\hline Lung cancer & 1 & $2.53(0.39,16.3 \mathrm{I})$ & NA & NA \\
\hline Gallbladder cancer & 1 & $1.39(0.92,2.11)$ & NA & NA \\
\hline ESCC & 1 & I.7I $(1.40,2.08)$ & NA & NA \\
\hline \multicolumn{5}{|l|}{ Venous invasion } \\
\hline Overall & 13 & $1.4 \mid(1.18,1.67)$ & $52.9 \%$ & 0.013 \\
\hline Colorectal cancer & 5 & $1.57(1.33,1.84)$ & $0.0 \%$ & 0.746 \\
\hline Gastric cancer & 3 & $1.48(1.04,2.12)$ & $35.6 \%$ & 0.212 \\
\hline Breast cancer & 1 & $0.69(0.42,1.11)$ & NA & NA \\
\hline EBDC & 1 & $0.95(0.61,1.49)$ & NA & NA \\
\hline Lung cancer & 1 & $3.16(0.50,19.87)$ & NA & NA \\
\hline Gallbladder cancer & 1 & $1.05(0.68,1.64)$ & NA & NA \\
\hline ESCC & 1 & $2.05(1.48,2.83)$ & NA & NA \\
\hline \multicolumn{5}{|l|}{ T stage } \\
\hline Overall & 18 & I.I4 (1.04, I.27) & $59.6 \%$ & 0.001 \\
\hline Colorectal cancer & 7 & $1.22(1.08,1.38)$ & $65.6 \%$ & 0.008 \\
\hline Gastric cancer & 4 & $1.04(0.85,1.28)$ & $29.7 \%$ & 0.234 \\
\hline Breast cancer & 2 & $0.66(0.3 \mathrm{I}, 1.40)$ & $0.0 \%$ & 0.895 \\
\hline EBDC & 1 & $1.13(0.79,1.62)$ & NA & NA \\
\hline Lung cancer & 1 & $0.83(0.66,1.04)$ & NA & NA \\
\hline Gallbladder cancer & 1 & $1.00(0.47,2.14)$ & NA & NA \\
\hline ESCC & 1 & $2.09(1.43,3.06)$ & NA & NA \\
\hline HNSCC & 1 & $0.91(0.71,1.17)$ & NA & NA \\
\hline \multicolumn{5}{|l|}{ N stage } \\
\hline Overall & 23 & $1.46(1.29,1.66)$ & $55.1 \%$ & 0.001 \\
\hline Colorectal cancer & 9 & $1.54(1.34,1.75)$ & $24.5 \%$ & 0.226 \\
\hline Gastric cancer & 4 & $1.28(1.11,1.47)$ & $0.0 \%$ & 0.393 \\
\hline Breast cancer & 3 & $1.46(1.04,2.04)$ & $41.6 \%$ & 0.180 \\
\hline Lung cancer & 3 & $2.00(0.44,8.97)$ & $80.2 \%$ & 0.006 \\
\hline EBDC & 1 & $1.06(0.57,1.97)$ & NA & NA \\
\hline
\end{tabular}


Table 3 (Continued)

\begin{tabular}{|c|c|c|c|c|}
\hline Subgroup & $\begin{array}{l}\text { Number } \\
\text { of studies }\end{array}$ & $\begin{array}{l}\text { Summary RR } \\
\text { (95\% Cls) }\end{array}$ & $I^{2}$ value & $\mathbf{P}_{\mathrm{h}}$ \\
\hline Gallbladder cancer & 1 & $1.13(0.56,2.29)$ & NA & NA \\
\hline ESCC & 1 & $2.70(1.98,3.68)$ & NA & NA \\
\hline HNSCC & 1 & $1.23(0.83,1.83)$ & NA & NA \\
\hline \multicolumn{5}{|l|}{$M$ stage } \\
\hline Overall & 14 & I.76 (I.34, 2.3I) & $42.1 \%$ & 0.049 \\
\hline Colorectal cancer & 5 & $1.47(1.15,1.87)$ & $9.2 \%$ & 0.354 \\
\hline Gastric cancer & 2 & $3.23(1.67,6.22)$ & $0.0 \%$ & 0.678 \\
\hline Lung cancer & 2 & $3.21(1.07,9.69)$ & $0.0 \%$ & 0.871 \\
\hline Breast cancer & I & $1.35(0.20,9.16)$ & NA & NA \\
\hline EBDC & 1 & $1.19(0.60,2.37)$ & NA & NA \\
\hline ESCC & I & $5.25(2.18,12.67)$ & NA & NA \\
\hline HNSCC & I & $0.89(0.39,2.05)$ & NA & NA \\
\hline OscC & I & $\mathrm{I} .24(0.70,2.21)$ & NA & NA \\
\hline \multicolumn{5}{|l|}{ Tumor stage } \\
\hline Overall & 15 & $1.42(1.19,1.68)$ & $69.9 \%$ & 0.000 \\
\hline Colorectal cancer & 8 & $\mathrm{I} .58(\mathrm{I} .36, \mathrm{I} .82)$ & $13.0 \%$ & 0.328 \\
\hline Gastric cancer & 2 & I.II $(0.88$, I.39) & $19.5 \%$ & 0.265 \\
\hline HNSCC & I & $1.08(0.79,1.49)$ & $58.7 \%$ & 0.120 \\
\hline Breast cancer & 1 & $0.90(0.27,2.97)$ & NA & NA \\
\hline EBDC & I & $1.12(0.74,1.70)$ & NA & NA \\
\hline ESCC & 1 & $3.04(1.95,4.73)$ & NA & NA \\
\hline
\end{tabular}

Note: $\mathrm{P}_{\mathrm{h}}$ : $P$-value for heterogeneity within each subgroup.

Abbreviations: RR, relative risk; $\mathrm{Cl}$, confidence interval; HNSCC, head and neck squamous cell carcinoma; OSCC, oral squamous cell carcinoma; EBDC, extrahepatic bile duct carcinoma; ESCC, esophageal squamous cell carcinoma; NA, not available.

of patients with gastric and colorectal cancer as a tumorassociated carbohydrate antigen, which was also proven by clinicopathological and immunohistochemical studies. ${ }^{42}$ The relationship between $\mathrm{sLe}^{\mathrm{X}}$ expression and cancer prognosis was identified by a number of studies, which did not show conformable results. To our knowledge, this is the first meta-analysis that systematically evaluates the relationship between $\mathrm{sLe}^{\mathrm{X}}$ expression and cancer prognosis and clinicopathology.

In the present study, a combined analysis of 29 articles (3,253 cancer patients) which showed the detection of high $\mathrm{sLe}^{\mathrm{X}}$ expression in tumor tissues with poor prognosis outcome in cancer patients was conducted. Our results indicated that $\mathrm{sLe}^{\mathrm{X}}$ expression was significantly correlated with lymphatic invasion, venous invasion, deep invasion ( $\mathrm{T}$ stage), lymph node metastasis ( $\mathrm{N}$ stage), distant metastasis ( $\mathrm{M}$ stage), tumor stage, tumor recurrence, and OS. On the other hand, although a high level of sLe $\mathrm{e}^{\mathrm{x}}$ expression was found in patients like the elderly, females, or patients with large size tumor and high differentiation, these results did not show any significance.

What makes $\mathrm{sLe}^{\mathrm{X}}$ overexpression account for the poor prognosis in cancer? By chemical analyses, it was shown that $\mathrm{sLe}^{\mathrm{X}}$ oligosaccharide was the minimal structure binding to E-, L-, and P-selectin, ${ }^{43}$ which was closely involved in the interaction between the endothelium and cancer cells.
$\mathrm{sLe}^{\mathrm{X}}$ is most commonly found in malignant tumors and plays a key role in cancer stem cell metastasis, hypoxia, and TNF- $\alpha$, and promotes tumor adhesion, invasion, and metastasis by upregulating the $\mathrm{sLe}^{\mathrm{X}}$ expression in the tumor microenvironment. ${ }^{44-46}$ In the present meta-analysis study, we also found that $\mathrm{sLe}^{\mathrm{X}}$ expression was correlated with tumor recurrence. On the other hand, it is widely accepted that expression of cell surface carbohydrates is altered during malignant transformation and tumor progression, and may influence determination of metastatic behavior of tumor cells. ${ }^{21,47}$ It has been identified that $\mathrm{sLe}^{\mathrm{X}}$ was a terminal tetrasaccharide moiety present on numerous membrane glycoproteins and glycolipids of epithelial and lymphatic cells. ${ }^{28}$ With such characters, a high level of $\mathrm{sLe}^{\mathrm{X}}$ contributes to cell adhesion, metastasis, and invasion because the cell surface antigens can combine with other cells directly. sLe $\mathrm{S}^{\mathrm{X}}$ in conjunction with mucins, promotes cellular motility, thus contributing to tumor cell spreading and metastasis. ${ }^{11,48}$ Furthermore, $\mathrm{sLe}^{\mathrm{X}}$ is expressed on granulocytes and monocytes which mediates inflammatory extravasation. ${ }^{49,50}$ However, the molecular biological mechanisms of how $\mathrm{sLe}^{\mathrm{X}}$ overexpression affects the cancer prognosis are complicated and still need further exploration. For the first time, our meta-analysis study revealed that $\mathrm{sLe}^{\mathrm{X}}$ could be a potential biomarker for poor cancer prognosis. 

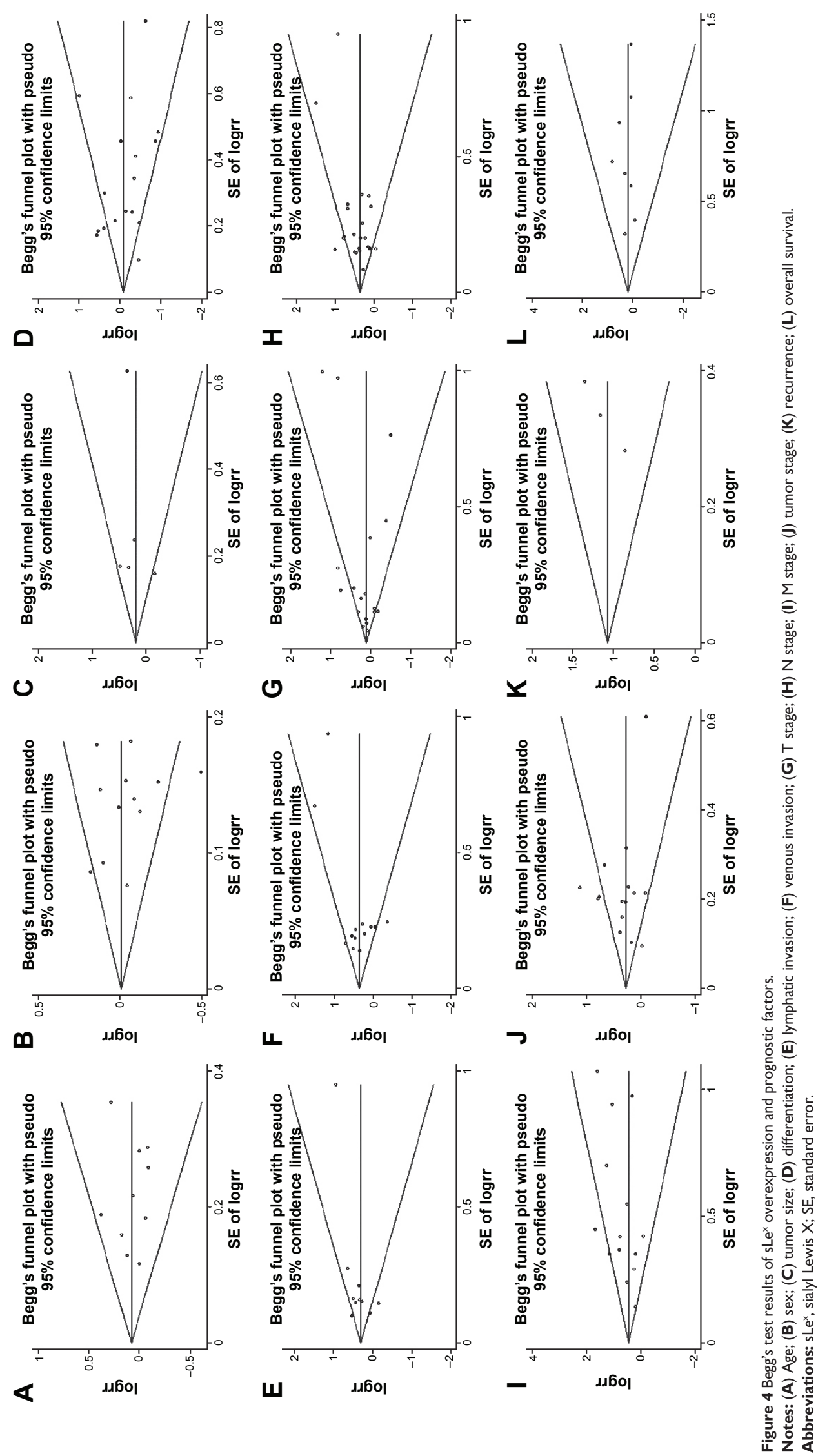
Due to the differences in nationality and cancer types which could cause heterogeneity among the studies, we conducted a subgroup analysis. In the subgroup analysis, the $\mathrm{SLe}^{\mathrm{X}}$ overexpression may play different roles caused by differentiation, venous invasion, $\mathrm{T}$ stage, $\mathrm{M}$ stage, tumor stage, and sex factors among different types of cancers. These factors contribute to the possible presence of heterogeneity between the studies. The difference might be owing to the molecular biological mechanisms of interactions between $\mathrm{SLe}^{\mathrm{x}}$ overexpression, and the occurrence and development of different types of cancers. Otherwise, ethnicity may be another factor that contributes to heterogeneity in sex, tumor size, differentiation, venous invasion, $\mathrm{T}$ stage, and $\mathrm{M}$ stage. It might be owing to the differences in genetic backgrounds and the environment among different races. We also found high heterogeneity in some subgroups, because biological behavior of cancer might be affected by many possible factors during the complicated process of tumor development.

Some limitations of this meta-analysis need to be acknowledged. First, all published studies and papers were written in English, some related published or unpublished studies that met the inclusion criteria were missed. Most of the studies reported positive results, while studies of negative results were all rejected. Second, some cancers such as oral squamous cell carcinoma, gallbladder cancer, pancreatic ductal adenocarcinoma, prostate cancer, and extrahepatic bile duct carcinoma were included in only one article respectively, so we could not evaluate pooled data in subgroup analyses. Third, all of the included studies had data of the $\mathrm{sLe}^{\mathrm{x}}$ expression which was detected by IHC methods. It might have some bias because of different antibodies and different standards of positive/negative $\mathrm{sLe}^{\mathrm{x}}$ expression. However, it was not available for us to do a subgroup analysis to analyze the underlying bias of IHC on the pooled odds ratios or HRs. Finally, multivariate analyses were not performed on OS data in most included studies, we calculated the pooled HR only from available HRs.

In conclusion, our meta-analysis showed that a high level of $\mathrm{sLe}^{\mathrm{X}}$ expression was significantly associated with lymphatic invasion, venous invasion, deep invasion, lymph node metastasis, distant metastasis, tumor stage, tumor recurrence, and $\mathrm{OS}$ in cancer. $\mathrm{sLe}^{\mathrm{X}}$ might be a new prognostic biomarker, and it might become a new diagnostic and therapeutic target for cancer. Further studies are required to explore the molecular biological mechanisms of $\mathrm{sLe}^{\mathrm{X}}$ and factors that caused significant heterogeneity in the present meta-analysis study.

\section{Acknowledgments}

This study was supported by National Natural Science Foundation of China (number 81001113). The authors are most grateful to all the participants in this study.

\section{Disclosure}

The authors declare no conflict of interest.

\section{References}

1. Pritchard-Jones K, Kaatsch P, Steliarova-Foucher E, Stiller CA, Coebergh JW. Cancer in children and adolescents in Europe: developments over 20 years and future challenges. Eur J Cancer. 2006;42(13): 2183-2190.

2. Fucic A, Gamulin M, Ferencic Z, et al. Environmental exposure to xenoestrogens and oestrogen related cancers: reproductive system, breast, lung, kidney, pancreas, and brain. Environ Health. 2012; 11 Suppl 1:S8.

3. Ferlay J, Steliarova-Foucher E, Lortet-Tieulent J, et al. Cancer incidence and mortality patterns in Europe: estimates for 40 countries in 2012. Eur J Cancer. 2013;49(6):1374-1403.

4. Sell S. Cancer-associated carbohydrates identified by monoclonal antibodies. Hum Pathol. 1990;21(10):1003-1019.

5. Dabelsteen E. Cell surface carbohydrates as prognostic markers in human carcinomas. J Pathol. 1996;179(4):358-369.

6. Liu S, Zhang Y, Liu Y, et al. FUT7 antisense sequence inhibits the expression of FUT7/sLeX and adhesion between embryonic and uterine cells. IUBMB Life. 2008;60(7):461-466.

7. Kannagi R, Kitahara A, Itai S, et al. Quantitative and qualitative characterization of human cancer-associated serum glycoprotein antigens expressing epitopes consisting of sialyl or sialyl-fucosyl type 1 chain. Cancer Res. 1988;48(13):3856-3863.

8. Itai S, Nishikata J, Takahashi N, et al. Differentiation-dependent expression of I and sialyl I antigens in the developing lung of human embryos and in lung cancers. Cancer Res. 1990;50(23):7603-7611.

9. Takada A, Ohmori K, Takahashi N, et al. Adhesion of human cancer cells to vascular endothelium mediated by a carbohydrate antigen, sialyl Lewis A. Biochem Biophys Res Commun. 1991;179(2):713-719.

10. Takada A, Ohmori K, Yoneda T, et al. Contribution of carbohydrate antigens sialyl Lewis A and sialyl Lewis X to adhesion of human cancer cells to vascular endothelium. Cancer Res. 1993;53(2):354-361.

11. Fukuoka K, Narita N, Saijo N. Increased expression of sialyl Lewis(x) antigen is associated with distant metastasis in lung cancer patients: immunohistochemical study on bronchofiberscopic biopsy specimens. Lung Cancer. 1998;20(2):109-116.

12. Ura H, Denno R, Hirata K, et al. Close correlation between increased sialyl-Lewisx expression and metastasis in human gastric carcinoma. World J Surg. 1997;21(7):773-776.

13. Davidson B, Gotlieb WH, Ben-Baruch G, et al. Expression of carbohydrate antigens in advanced-stage ovarian carcinomas and their metastases-A clinicopathologic study. Gynecol Oncol. 2000;77(1): 35-43.

14. Kurahara S, Shinohara M, Ikebe T, et al. Immunohistochemical study of sialyl Le(a) and sialyl Le(x) antigen in oral squamous cell carcinoma: the association of sialyl Le(a) expression with the metastatic potential. Head Neck. 1999;21(4):330-337.

15. Schiffmann L, Schwarz F, Linnebacher M, et al. A novel sialyl Le(X) expression score as a potential prognostic tool in colorectal cancer. World J Surg Oncol. 2012;10:95.

16. Nakagoe T, Fukushima K, Nanashima A, et al. Expression of Lewis(a), sialyl Lewis(a), Lewis(x) and sialyl Lewis(x) antigens as prognostic factors in patients with colorectal cancer. Can J Gastroenterol. 2000; 14(9):753-760. 
17. Baldus SE, Zirbes TK, Monig SP, et al. Histopathological subtypes and prognosis of gastric cancer are correlated with the expression of mucinassociated sialylated antigens: Sialosyl-Lewis(a), sialosyl-Lewis(x) and sialosyl-Tn. Tumor Biol. 1998;19(6):445-453.

18. Nakamori S, Kameyama M, Imaoka S, et al. Increased expression of sialyl Lewisx antigen correlates with poor survival in patients with colorectal carcinoma: clinicopathological and immunohistochemical study. Cancer Res. 1993;53(15):3632-3637.

19. Yamaguchi A, Saitoh M, Goi T, et al. Sialyl-lewis-x antigen immunoreaction of colorectal-cancer and its relationship to hematogenous metastasis. Oncol Rep. 1994;1(4):731-734.

20. Idikio HA. Sialyl-Lewis-X, Gleason grade and stage in non-metastatic human prostate cancer. Glycoconj J. 1997;14(7):875-877.

21. Nakamori S, Kameyama M, Imaoka S, et al. Involvement of carbohydrate antigen sialyl Lewis(x) in colorectal cancer metastasis. Dis Colon Rectum. 1997;40(4):420-431.

22. Shimodaira K, Nakayama J, Nakamura N, et al. Carcinoma-associated expression of core 2 beta-1,6-N-acetylglucosaminyltransferase gene in human colorectal cancer: role of O-glycans in tumor progression. Cancer Res. 1997;57(23):5201-5206.

23. Farmer RW, Richtsmeier WJ, Scher RL. Identification of sialyl Lewis-x in squamous cell carcinoma of the head and neck. Head Neck. 1998;20(8):726-731.

24. Tatsumi M, Watanabe A, Sawada H, et al. Immunohistochemical expression of the sialyl Lewis $\mathrm{x}$ antigen on gastric cancer cells correlates with the presence of liver metastasis. Clin Exp Metastasis. 1998;16(8): 743-750.

25. Yamaguchi A, Ding K, Maehara M, Goi T, Nakagawara G. Expression of nm23-H1 gene and Sialyl Lewis X antigen in breast cancer. Oncology. 1998;55(4):357-362.

26. Takao S, Uchikura K, Yonezawa S, Shinchi H, Aikou T. Mucin core protein expression in extrahepatic bile duct carcinoma is associated with metastases to the liver and poor prognosis. Cancer. 1999;86(10): 1966-1975.

27. Futamura N, Nakamura S, Tatematsu M, et al. Clinicopathologic significance of sialyl Le(x) expression in advanced gastric carcinoma. Br J Cancer. 2000;83(12):1681-1687.

28. Grabowski P, Mann B, Mansmann U, et al. Expression of SIALYL-Le(x) antigen defined by MAb AM-3 is an independent prognostic marker in colorectal carcinoma patients. Int J Cancer. 2000;88(2):281-286.

29. Machida E, Nakayama J, Amano J, Fukuda M. Clinicopathological significance of core 2 beta1,6-N-acetylglucosaminyltransferase messenger RNA expressed in the pulmonary adenocarcinoma determined by in situ hybridization. Cancer Res. 2001;61(5):2226-2231.

30. Takahashi S, Oda T, Hasebe T, et al. Overexpression of sialyl Lewis $x$ antigen is associated with formation of extratumoral venous invasion and predicts postoperative development of massive hepatic metastasis in cases with pancreatic ductal adenocarcinoma. Pathobiology. 2001; 69(3):127-135.

31. Baldus SE, Monig SP, Hanisch FG, et al. Comparative evaluation of the prognostic value of MUC1, MUC2, sialyl-Lewisa and sialyl-lewisx antigens in colorectal adenocarcinoma. Histopathology. 2002;40(5): 440-449.

32. Konno A, Hoshino Y, Terashima S, Motoki R, Kawaguchi T. Carbohydrate expression profile of colorectal cancer cells is relevant to metastatic pattern and prognosis. Clin Exp Metastasis. 2002;19(1):61-70.
33. Nakagoe T, Fukushima K, Itoyanagi N, et al. Expression of $\mathrm{ABH} /$ Lewis-related antigens as prognostic factors in patients with breast cancer. J Cancer Res Clin Oncol. 2002;128(5):257-264.

34. Nakagoe T, Fukushima K, Sawai T, et al. Increased expression of sialyl Lewis(x) antigen as a prognostic factor in patients with stage 0 , I, and II gastric cancer. Cancer Lett. 2002;175(2):213-221.

35. Kashiwagi H, Kijima H, Dowaki S, et al. Clinicopathological significance of sialyl Lex expression in human gallbladder carcinoma. Oncol Rep. 2004;11(6):1139-1143.

36. Yu CJ, Shih JY, Lee YC, et al. Sialyl Lewis antigens: association with MUC5AC protein and correlation with post-operative recurrence of non-small cell lung cancer. Lung Cancer. 2005;47(1):59-67.

37. Faried A, Kimura H, Faried LS, et al. Expression of carbohydrate antigens in human esophageal squamous cell carcinoma: Prognostic application and its diagnostic implications. Ann Surg Oncol. 2007;14(2) 960-967.

38. Croce MV, Rabassa ME, Pereyra A, Segal-Eiras A. Differential expression of MUC1 and carbohydrate antigens in primary and secondary head and neck squamous cell carcinoma. Head Neck. 2008;30(5): 647-657.

39. Sozzani P, Arisio R, Porpiglia M, Benedetto C. Is Sialyl Lewis x antigen expression a prognostic factor in patients with breast cancer? Int J Surg Pathol. 2008;16(4):365-374.

40. Portela SV, Martin CV, Romay LM, et al. sLea and sLex expression in colorectal cancer: implications for tumourigenesis and disease prognosis. Histol Histopathol. 2011;26(10):1305-1316.

41. Siegel R, Naishadham D, Jemal A. Cancer statistics, 2013. CA Cancer J Clin. 2013;63(1):11-30.

42. Nakagoe T, Ishikawa H, Nakao H, et al. [Sialyl Lewis(x)-I (SLX) as a tumor-associated carbohydrate antigen in sera in patients with gastric and colorectal cancer - evaluation according to clinico-pathological factors]. Gan To Kagaku Ryoho. 1989;16(4 Pt 1):819-825. Japanese.

43. Foxall C, Watson SR, Dowbenko D, et al. The three members of the selectin receptor family recognize a common carbohydrate epitope, the sialyl-Lex oligosaccharide. J Cell Biol. 1992;117(4):895-902.

44. Koike T, Kimura N, Miyazaki K, et al. Hypoxia induces adhesion molecules on cancer cells: A missing link between Warburg effect and induction of selectin-ligand carbohydrates. Proc Natl Acad Sci US A. 2004;101(21):8132-8137.

45. St Hill CA, Krieser K, Farooqui M. Neutrophil interactions with sialyl Lewis $\mathrm{X}$ on human nonsmall cell lung carcinoma cells regulate invasive behavior. Cancer. 2011;117(19):4493-4505.

46. Desiderio V, Papagerakis $\mathrm{P}$, Tirino V, et al. Increased fucosylation has a pivotal role in invasive and metastatic properties of head and neck cancer stem cells. Oncotarget. 2015;6(1):71-84.

47. Irimura T, Reading CL. Surface properties of metastatic tumor cells. Cancer Bull. 1987;39(3):132-141.

48. Byrd JC, Bresalier RS. Mucins and mucin-binding proteins in colorectal cancer. Cancer Metastasis Rev. 2004;23(1-2):77-99.

49. Sperandio M. Selectins and glycosyltransferases in leukocyte rolling in vivo. FEBS J. 2006;273(19):4377-4389.

50. Zimmerman BJ, Paulson JC, Arrhenius TS, Gaeta FC, Granger DN. Thrombin receptor peptide-mediated leukocyte rolling in rat mesenteric venules: roles of P-selectin and sialyl Lewis X. Am J Physiol. 1994; 267(3 Pt 2):H1049-H1053.
OncoTargets and Therapy

\section{Publish your work in this journal}

OncoTargets and Therapy is an international, peer-reviewed, open access journal focusing on the pathological basis of all cancers, potential targets for therapy and treatment protocols employed to improve the management of cancer patients. The journal also focuses on the impact of management programs and new therapeutic agents and protocols on

\section{Dovepress}

patient perspectives such as quality of life, adherence and satisfaction. The manuscript management system is completely online and includes a very quick and fair peer-review system, which is all easy to use. Visit http://www.dovepress.com/testimonials.php to read real quotes from published authors. 